\title{
Diffusive dynamics of elongated particles in active colloidal suspensions of motile algae
}

\author{
Florian von Rüling ${ }^{1} \cdot$ Francine Kolley $^{1,2} \cdot$ Alexey Eremin $^{1}$ (CD
}

Received: 31 January 2020 / Revised: 26 March 2020 / Accepted: 7 April 2020 / Published online: 3 June 2020

(C) The Author(s) 2020

\begin{abstract}
Swimming microorganisms can influence the diffusion of passive particles. The effect of this swimmer-particle interaction depends on different properties, such as the hydrodynamic field of the swimmer and the relative sizes of microorganisms and particles. We investigated an enhancement of the diffusion of silica doublets in a suspension of microalgae Chlamydomonas reinhardtii in a flat capillary. Depending on the concentration of microswimmers, the translational and rotational diffusion constants increase by several orders of magnitude in the presence of the swimming algae. For low concentrations of algae, the doublets exhibit Brownian motion in a fluctuating flow field generated by multiple swimmers. One can observe strong, diffusive transport caused by occasional large displacements. At high swimmer concentration, the algae form dense clusters, where the rotational motion of the doublets shows a subdiffusive behaviour while the translational motion remains diffusive.
\end{abstract}

Keywords Active matter $\cdot$ Microswimmers $\cdot$ Diffusion

\section{Introduction}

Swimming microorganisms like the motile microalgae Chlamydomonas reinhardtii dwell in complex liquidinfused environments, where they encounter suspended or sedimented immersed passive particles. The diffusive motion of the particles can be enhanced by self-propelled swimmers due to hydrodynamic interactions [1-3] and collisions [4]. Thus, swimming microorganisms can accomplish fluid mixing on the microscale. As a result, small selfpropelled objects become crucial for the transport processes in their environment. It was demonstrated, that the flow generated by flagellar beating is necessary for sufficient nutrient uptake and waste removal of certain multicellular organisms [5]. However, the question whether the biogenic contribution to mixing is significant is controversial

Florian von Rüling

florian.rueling@st.ovgu.de

1 Institute of Physics, Otto von Guericke University Magdeburg, Magdeburg, 39106, Germany

2 Present address: Center for Advancing Electronics (cfaed), TU Dresden, Dresden, 01069, Germany even for macroscopic swimmers like centimetre-sized krill [6].

The investigation of interactions of microswimmers and passive objects is of great interest from a physicist's perspective. A fluid containing self-propelled objects is out of thermodynamic equilibrium due to the energy input at the level of the single swimmers [7]. Thus, a passive particle immersed in such an active fluid is an experimental model system of nonequilibrium statistical mechanics [8]. The athermal hydrodynamic and steric contributions to the particle transport can result in intriguing phenomena like the anomalous diffusion of anisometric objects [9].

Furthermore, the employment of swimmer-particle interactions in microfluidic applications is auspicious. Experimental research suggests that microswimmers can serve as actuators [10] or, exploiting taxes of biological swimmers, accomplish asymmetric mixing [11].

The enhancement of passive particle diffusion occurs for a variety of swimmer-particle combinations $[2-4,9,12]$. Yet, the details depend on the swimmer hydrodynamics [9, 12] and the particle size [13]. In previous experiments the motion of swimmers was confined to quasi-2D [2, 4, 9, 12].

In this paper, we report an experimental study on the motion of sedimented elongated passive particles in the presence of Chlamydomonas reinhardtii in a threedimensional fluid. 


\section{Materials and methods}

\section{Materials}

Wild-type Chlamydomonas reinhardtii of strain SAG 1132a were purchased from the Culture Collection of Algae at Göttingen University. The microalgae were grown axenically in tris-acetate-phosphate medium (TAP) (see Refs. [14, 15]) with air bubbling on a 14-h-10-h day-night cycle. The strain was maintained by weekly sub-culturing.

Silica beads (MicroSil Microspheres SS06N from Bangs Laboratories, Inc.; mean diameter, $4.89 \mu \mathrm{m}$ ) were used as passive particles. Beads were washed in distilled water multiple times using a centrifuge and then stored in a fridge. The spheres were heated to room temperature and redispersed prior to adding them to the suspension of algae. Occasionally, elongated objects consisting of two beads were found (see Fig. 1c). The maximal axial length of such silica doublets (about $10 \mu \mathrm{m}$ ) is comparable with the diameter of Chlamydomonas reinhardtii $(5 \mu \mathrm{m}-10 \mu \mathrm{m})$. The algae were re-dispersed in distilled water to reduce sticking of the silica beads to the glass. Polyethyleneglycol (PEG; purchased from Sigma-Aldrich, typical $M_{v}$ approx. $900,000)$ was added to the final concentration of $0.1 \mathrm{wt} \%$ of PEG.

Samples were observed in silanized rectangular glass capillaries (Rectangle Boro Tubing VitroTubes from CM Scientific; thickness, $500 \mu \mathrm{m}$; width, $5 \mathrm{~mm}$; length, several centimetres). The silanization procedure, which additionally avoids sticking of Chlamydomonas to glass, was based on a method described in Ref. [16].

\section{Methods}

A sketch of the experimental setup is provided in Fig. 1a. Active colloidal suspensions of Chlamydomonas reinhardtii were observed using an inverted microscope (Zeiss Observer.D1). Schott KL 2500 LCD cold light source

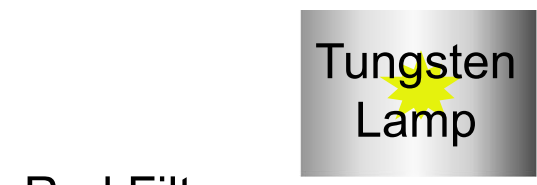

Red Filter

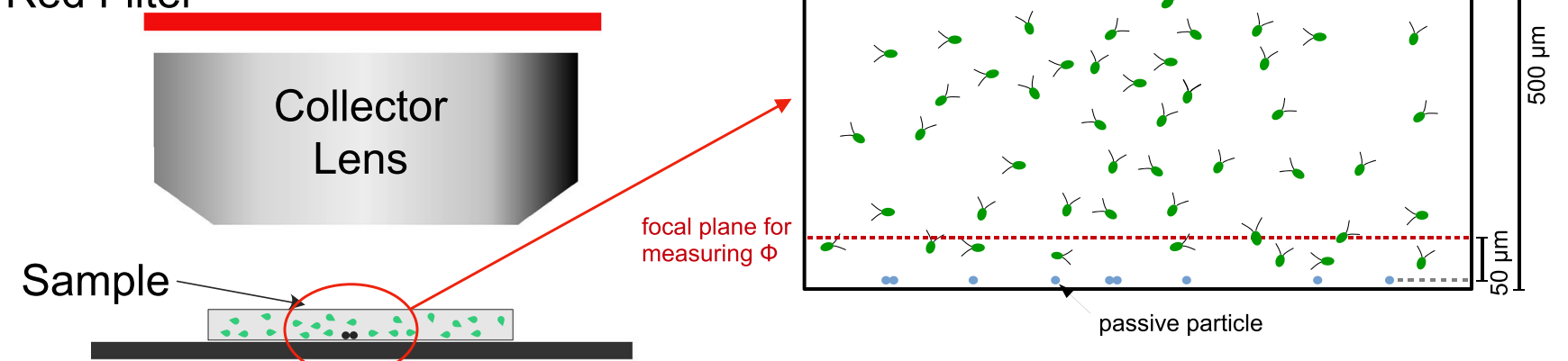

(a)

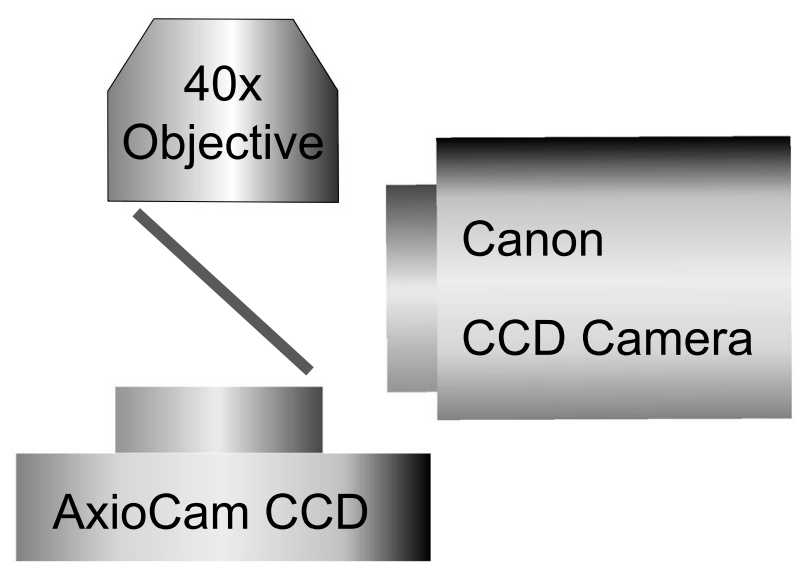

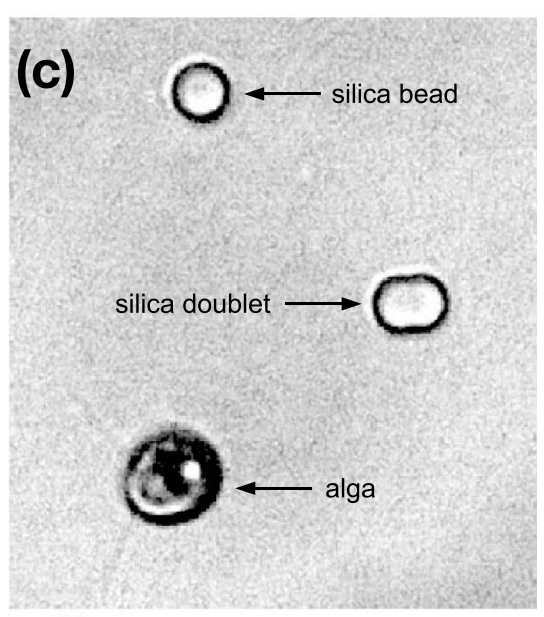

$\underline{10 \mu \mathrm{m}}$
Fig. 1 Experimental setup and conditions. a Scheme of the experimental setup based on the inverted microscope stand. b Schematic side view of a capillary with motile algae and sedimented passive particles.
The dashed red line represents the focal plane of videos recorded to measure the area fraction of swimming algae $\Phi$. c Micrograph of a Chlamydomonas reinhardtii cell and passive silica particles 
with a red longpass filter $(\lambda \geq 630 \mathrm{~nm}$ ) was used for the ambient illumination of the cells to suppress phototaxis. The sample was also illuminated under microscope with a halogen lamp (Zeiss HAL 100) through a longpass filter (light intensity $\approx 30 \mathrm{~W} / \mathrm{m}^{2}$, wavelength $\lambda \geq 650 \mathrm{~nm}$ ). The cells were kept for $30 \mathrm{~min}$ on the microscope stage before data acquisition to allow the algae to adopt to the illumination conditions. The motion of passive particles was recorded using $\times 40$ magnification (Objective: Zeiss LD Plan-NEOFLUAR $\times$ 40/0.6 korr) and AxioCam HRc (Carl Zeiss $\mathrm{GmbH}$ ) $\mathrm{CCD}$ camera (1-5 fps frame rate, and the resolution of $1388 \times 1040)$. Typical acquisition times were $30 \mathrm{~min}$ and $10 \mathrm{~min}$. To investigate silica particles without swimmers, we used the acquisition framerate, $0.2 \mathrm{fps}$ and acquisition time, $90 \mathrm{~min}$.

To characterise the fast dynamics of microswimmers interacting with the silica particles, videos of Chlamydomonas reinhardtii in a thin fluid layer located about $50 \mu \mathrm{m}$ above the doublets (see Fig. 1b) were acquired at a magnification of $\times 20$ (objective: Zeiss LD PlanNEOFLUAR $\times$ 20/0.4 corr) with a Canon EOS 600D CCD camera (acquisition framerate, circa $60 \mathrm{fps}$; resolution, $1280 \times 720)$. The videos were taken before and after acquiring the $\times 40$ videos showing the motion of passive particles. Using a particle tracking algorithm, the area fraction of swimming algae was extracted from those videos. Area fractions of clustering algae were measured in the same way in the plane of the doublets. When dense clusters of algae occurred, the number of passive objects in this plane can be neglected compared with the cell number.

Theoretical values for the diffusion of passive particles in a thermal bath were calculated for comparison with experimental values using equations for cylindrical particles [17]:

$$
\begin{aligned}
D_{\mathrm{r}} & =\frac{3 k_{B} T}{\pi \eta L^{3}} \cdot\left(\ln p+\delta_{\perp}\right) \\
D_{\|} & =\frac{k_{B} T}{2 \pi \eta L} \cdot\left(\ln p+v_{\|}\right) \\
D_{\perp} & =\frac{k_{B} T}{4 \pi \eta L} \cdot\left(\ln p+v_{\perp}\right)
\end{aligned}
$$

where $D_{\mathrm{r}}$ is the rotational diffusion coefficient; $D_{\|, \perp}$ are the translational diffusion constants for motion along the major and minor axis of the particle, respectively; $L$ is the length and $p$ is the aspect ratio of the particles which are immersed in a fluid of viscosity $\eta$ at a temperature $T . k_{B}$ is the Boltzmann constant. $\delta_{\perp}, v$ are given in [17]:

$$
\begin{aligned}
\delta_{\perp} & =-0.662+\frac{0.917}{p}-\frac{0.05}{p^{2}} \\
\nu_{\|} & =-0.207+\frac{0.98}{p}-\frac{0.133}{p^{2}} \\
\nu_{\perp} & =0.839+\frac{0.185}{p}+\frac{0.233}{p^{2}}
\end{aligned}
$$

In our system, the motion of passive particles is restricted to two dimensions and the translational diffusion coefficient $D_{\mathrm{t}}$ can be obtained using Eqs. (2) and (3)

$D_{\mathrm{t}}=\frac{D_{\|}+D_{\perp}}{2}$

Substituting Eqs. (4)-(6) into Eqs. (1)-(3) and (7) with the rod length $L=10 \mu \mathrm{m}$, aspect ratio $p=2$, fluid temperature $T=293 \mathrm{~K}$ and fluid viscosity $\eta=1.76 \mathrm{mPas}$ (viscosity of distilled water with $0.1 \mathrm{wt} . \%$ PEG measured in [18]), we obtain the following $D_{\mathrm{r}} \approx 1.0 \cdot 10^{-3} \mathrm{rad}^{2} / \mathrm{s}$, $D_{\mathrm{t}} \approx 3.3 \cdot 10^{-2} \mu \mathrm{m}^{2} / \mathrm{s}$.

\section{Results and discussion}

\section{Interaction of freely swimming Chlamydomonas reinhardtii with passive particles}

We investigated the motion of silica doublets in an active bath of Chlamydomonas reinhardtii for different swimmer concentrations. As a measure of the concentration of active swimmers, we used the area fraction of moving algae $\Phi$ measured close to the lower wall of the capillary (approximately $50 \mu \mathrm{m}$ above the wall, see Fig. 1b) rather than the volume fraction. $\Phi$ was varied from $\Phi=0 \%$ to $\Phi \approx 8.6 \%$, the latter corresponds to a cell number density of approximately $1.02 \cdot 10^{8} \mathrm{ml}^{-1}$ or a volume fraction of about $5.4 \%$.

Note that the silica particles, having a higher density than the surrounding medium, sink to the bottom of the glass capillary. Thus, they are located close to a solid boundary. The experimental values for the diffusion coefficients of a doublet in a the absence of swimmers read: $D_{\mathrm{r}}=(5.3 \pm$ $0.3) \cdot 10^{-4} \mathrm{rad}^{2} / \mathrm{s}, D_{\mathrm{t}}=(5.3 \pm 0.4) \cdot 10^{-4} \mu \mathrm{m}^{2} / \mathrm{s}$.

In order to compare the experimental and the theoretical values for the diffusion constants of passive particles near the wall, Faxén's correction, considering the hydrodynamic interaction with the wall, must be taken into account [19]. The effect of the solid boundary was studied for a sphere of equivalent volume using the Einstein relation with drag coefficients corrected according to [19]:

$$
\begin{aligned}
& \gamma=\frac{\gamma_{0}}{1-\frac{9}{16} \cdot \frac{R}{h}+\frac{1}{8} \cdot\left(\frac{R}{h}\right)^{3}} \\
& \beta=\frac{\beta_{0}}{1-\frac{1}{8} \cdot\left(\frac{R}{h}\right)^{3}}
\end{aligned}
$$

where $R$ is the radius of the sphere and $h$ the distance between the centre of the sphere and the wall. For $h \rightarrow R$, the correction yields a change of $44 \%$ for the translational and $13 \%$ for the rotational diffusion constant. This value is too small to explain the discrepancy between measured 
and predicted values. Therefore, this difference between the experiment and the theory can be attributed to the adhesion of particles to the capillary wall. In addition, the deviation between the values is much more pronounced for translational than for rotational motion. This can be seen as an indication that the particles are able to rotate easier about the contact area. At the same time, the translational displacement is hindered.

The motion of algae significantly enhances the positional and orientational fluctuations of the passive particles. The magnitude of the particle displacement in a fixed time interval is shown in Fig. 2 for low swimmer concentration. Long periods of relatively small particle displacements are interrupted by distinct peaks that signal strong translations. Those peaks correspond to either contact interactions or the hydrodynamic disturbances caused by the swimmers in the close vicinity of the particles. During the periods of small particle displacement, there are no algae in the vicinity of the particle which then is subjected to a collective hydrodynamic field that is the superposition of the far fields the swimmers generate. This flow field is fluctuating since swimmers translate and reorient, thus causing effective Brownian motion of the passive particle. Note that during the periods of small displacement, between the collisions, the diffusive motion is already significantly enhanced with respect to purely thermodynamical diffusion. As the swimmer concentration is increased, the frequency of the large displacement events grows until the periods of small displacement vanish. Owing to the interactions with swimming algae, both rotational and translational diffusion constants are significantly enhanced in the active bath of Chlamydomonas reinhardtii.

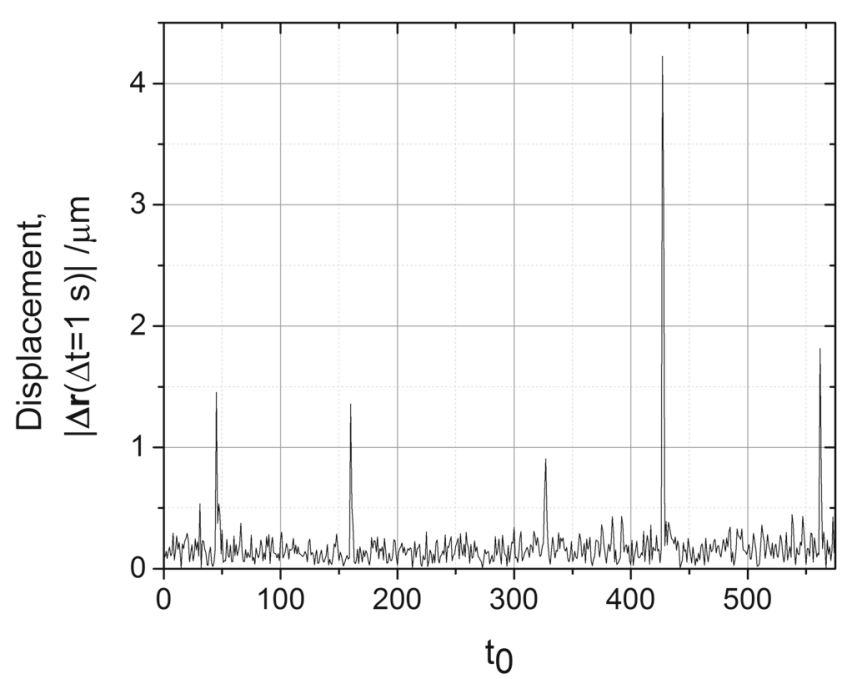

Fig. 2 Absolute value of the displacement $\left|\mathbf{r}\left(t_{0}+\Delta t\right)-\mathbf{r}\left(t_{0}\right)\right|$ of a silica doublet in a dilute suspension of Chlamydomonas reinhardtii (area fraction $\Phi \approx 0.3 \%$ ) in the time interval $\Delta t=1 \mathrm{~s}$ for different starting points $t_{0}$
The rotational diffusion constant $D_{\mathrm{r}}(\Phi)$ is shown for different values of the area fraction of moving algae $\Phi$ in Fig. 3. With increasing concentration of the microswimmers, $D_{\mathrm{r}}$ changes by nearly three orders of magnitude. The dependence of $D_{\mathrm{r}}(\Phi)$ is linear for low $\Phi$, which is in agreement with theory by Pushkin et al. [20]. Above $\Phi \approx 3.9 \%$ the behaviour becomes nonlinear $D_{\mathrm{r}}(\Phi) \propto \Phi^{n}$ with $n>1.9$. At this volume fraction, the motion of algae remains only weakly correlated. Thus, the crossover at $3.9 \%$ cannot be explained by swarming behaviour as in [9]. The nonlinearity can arise due to the simultaneous action of the advective flow fields of multiple swimmers on the particle [2].

The translational diffusion constant $D_{\mathrm{t}}$ increases by almost four orders of magnitude in the presence of algae as compared with the case of purely thermodynamical diffusion. Intriguingly, the dependence of $D_{\mathrm{t}}$ on $\Phi$ is nonlinear even at low $\Phi<3.9 \%$ (see Fig. 4). However, $D_{\mathrm{t}}$ remains proportional to $\Phi^{n}$ with $n \approx 1.7$. The unexpectedly strong dependence of the translational diffusion on the number of swimmers is apparently related to the contact adhesion of the passive particles to the capillary wall, which suppresses the linear regime at low area fraction $\Phi<3.9 \%$. Experiments without swimmers imply that the passive particles can not diffuse freely which is seemingly related to the partial contact of silica particles to the glass wall of the capillary. Yet, algae provide much more energy than the thermally fluctuating fluid and promote particles to overcome the contact adhesion. This situation is different from the rotational diffusion, which is less hindered by the contact to the wall.

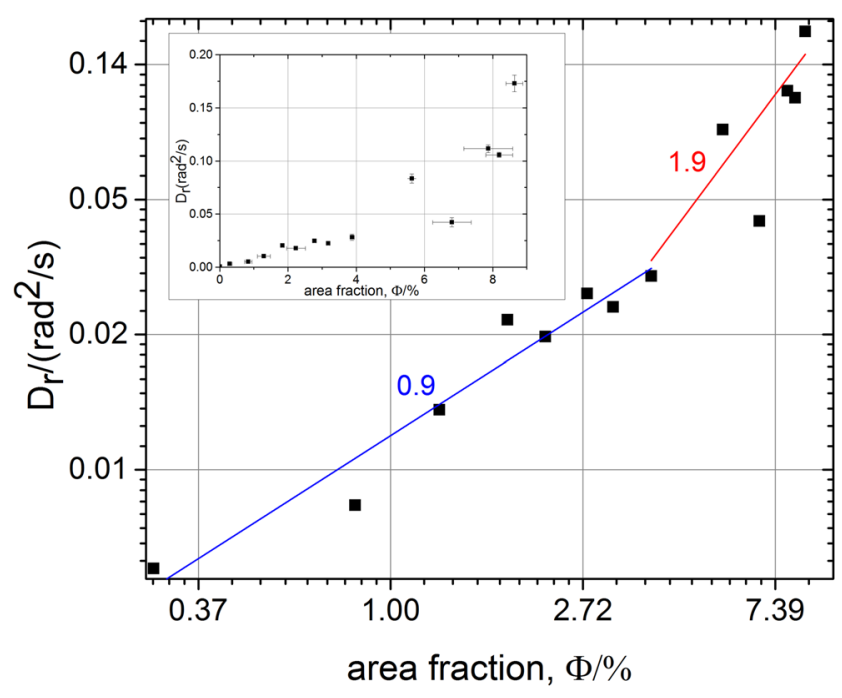

Fig. 3 The rotational diffusion constant $D_{\mathrm{r}}$ of elongated silica particles in active baths with different area fractions $\Phi$ of motile Chlamydomonas reinhardtii. The blue and red numbers denote the slope of the linear fits in the double-logarithmic scaling for low and high concentrations of algae, respectively. Linear scaling is shown in the inset 


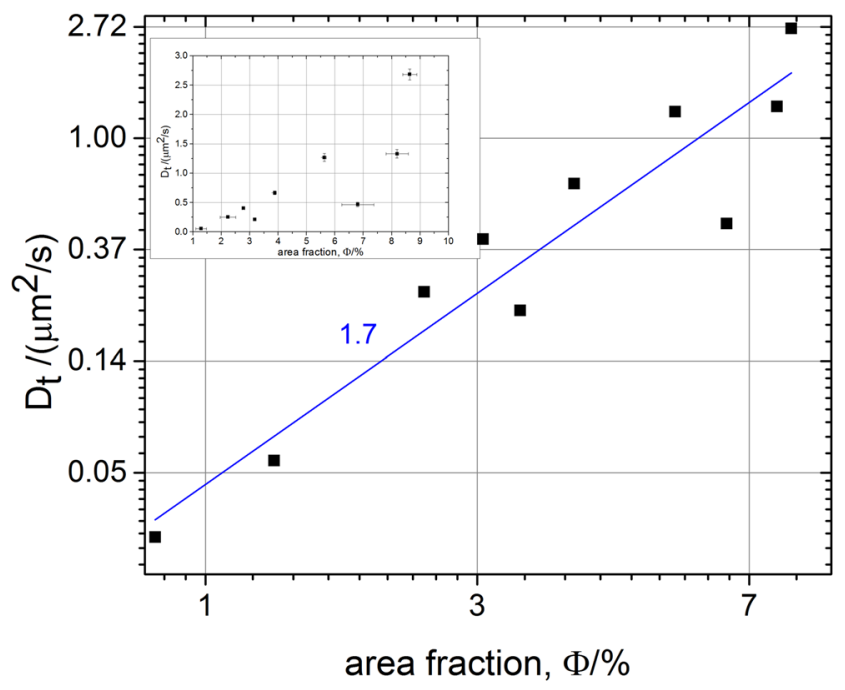

Fig. 4 The translational diffusion constant $D_{\mathrm{t}}$ of elongated silica particles in active baths with different area fractions of motile Chlamydomonas reinhardtii $\Phi$. The number next to the linear fit in the double-logarithmic plot denotes the slope. Linear scaling is shown in the inset

\section{Motion of passive particles in a clustered state}

Algae tend to adhere to the lower wall of the glass capillary during the experiments. The number of such adhered algae can be strongly reduced by using a suitable illumination in the red band of the visible light spectrum [21]. However, at high swimmer concentrations (above $\Phi \approx 9 \%$ ), the surface layer becomes rapidly populated with the algae whose motion becomes strongly restricted by the neighbours and the swimmers in the bulk. There is an exchange between the surface layer and the bulk, which occurs on the time scale of several minutes. Above the layer of sedimented algae a high number of swimmers (comparable with the case with $\Phi \approx 8.6 \%$ ) contributes to the active bath. At the same time, the area fraction at the surface layer reaches about $33 \%$. Figure 5 a shows an example of such a situation, where the lower capillary wall is densely covered with active but restricted in motion algae. Positional correlations become stronger pronounced and the motion of the algae appears caged. However, in this case, the correlations are only short-ranged and short-living (Fig. 5b). The motion of the passive particles in such a crowded environment becomes obstructed (Fig. 5c).

Due to the sedimentation, the number of algae in the clustered state exhibits a complex dependence on the total number of algae in the capillary. Sparser clusters, covering about $15 \%$ of the bottom wall area, formed in the case with the largest cell number of Chlamydomonas reinhardtii. Note that for the cases with clustering algae the area fractions are only estimates since the accuracy is reduced by the complex background of the videos.
Local ordering of algae can be characterised by the pair correlation function which shows positional correlations of the cells. Since the system is isotropic, it is sufficient to study correlations in dependence on the distance. The (momentary) radial pair correlation function $g_{2}(r)=$ $\left\langle n\left(r_{0}\right) n\left(r_{0}+r\right)\right\rangle_{r_{0}}$ of Chlamydomonas reinhardtii-cells in the denser clustered state is shown in Fig. $5 b$. Here, $n$ is the number of cells and $r$ is a radial coordinate. The function $n(r)$ was obtained by cell detection and counting the number of cells in a circular segment of radius $r$ centred around a selected cell (see Ref. [22]). The pair correlation function has pronounced first and second maxima indicating short correlations between the algae forming small clusters. The neighbouring cells appear often in contact.

Figure $6 \mathrm{a}$ and $\mathrm{b}$ show the trajectories of algae in the denser and sparser clustered state, respectively. The trajectory in the dense state terminates when the alga swims away. A cell is confined for minutes and can traverse the clusters of algae only slowly, its trajectory reaching an extension of about $40 \mu \mathrm{m}$ in more than $4 \mathrm{~min}$. The path of the alga in the sparser clustered state with a strong active bath (Fig. 6b) spans more than $50 \mu \mathrm{m}$, five times the body length of Chlamydomonas reinhardtii, in about $70 \mathrm{~s}$. If the algae were adhered to the wall with their about $12 \mu \mathrm{m}$ [23] long flagella, their centre of mass motion would be restricted to a circle with diameter $34 \mu \mathrm{m}$ under the best conditions. Practically, if the clustering algae were adhered, this distance would be reduced due to the entanglement of flagella. Thus, algae in the clustered state are not adhered to the capillary wall. The velocities of the clustering algae are one order of magnitude smaller than bulk self-propulsion velocities. A mean squared displacement analysis does not evince any ballistic regime. Yet, the time resolution of the experiments was only about $5 \mathrm{fps}$ and the motion of algae in the clustered state can be strongly randomised due to interactions with the fast algae of the active bath. For an alga in the sparser clustered state, the translational diffusion coefficient $D_{\mathrm{t}}^{\text {Alga }} \approx 3.6 \mu \mathrm{m}^{2} / \mathrm{s}$ can be determined, which exceeds the translational diffusion coefficients measured for the silica doublets of similar size.

A passive particle enclosed by clustering algae is driven due to the interactions with algae from the bath and the clusters. However, the clusters also provide a dynamic geometrical constraint: Algae can jam the particle and even form cages around it. The trajectory of a silica doublet surrounded by algae in the dense clustered state is displayed in Fig. 5c and exhibits intermittent confinement. The translational diffusion constant of this particle in this state takes only a moderate value of $D_{\mathrm{t}} \approx 0.5 \mu \mathrm{m}^{2} / \mathrm{s}$ and its rotational motion even becomes subdiffusive on a time scale of $10 \mathrm{~s}$. Since algae and passive particles are of comparable size, a single alga in the vicinity of the 


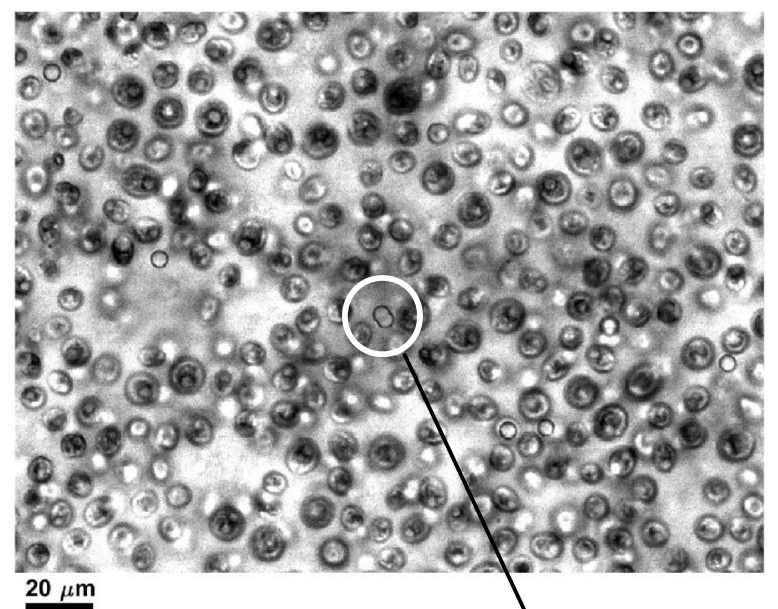

(a)
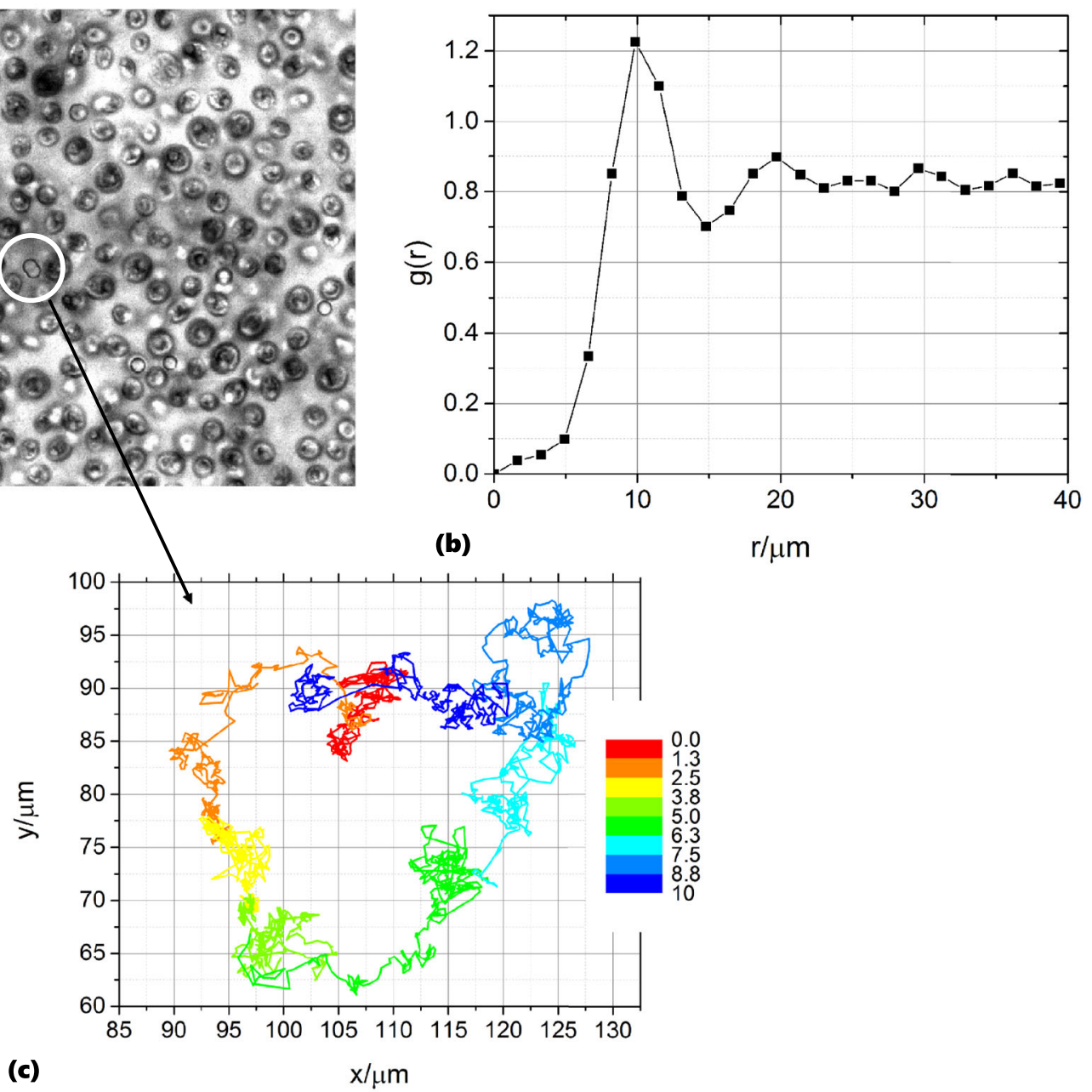

(b)

$\mathrm{r} / \mu \mathrm{m}$

(exemplary) of Chlamydomonas reinhardtii in the clustered state. c

Fig. 5 a At high cell concentrations, Chlamydomonas reinhardtii densely cover the lower capillary wall. Besides the microalgae, few silica particles can be seen. b Radial pair correlation function The trajectory of a silica doublet enclosed by clusters of Chlamydomonas reinhardtii. The colour indicates the time in minutes
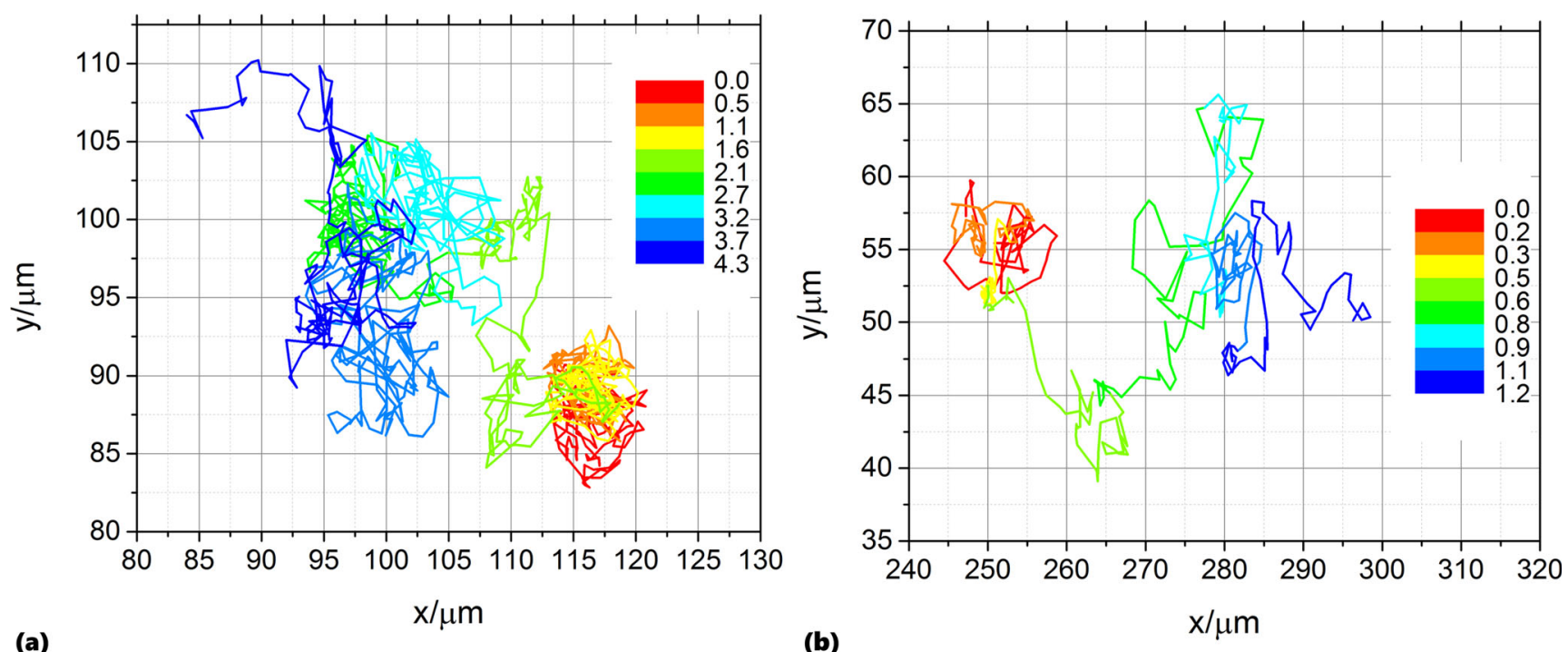

Fig. 6 Trajectory of a Chlamydomonas reinhardtii-alga in the a denser and $\mathbf{b}$ sparser clustered state. The colour coding indicates the time in minutes 


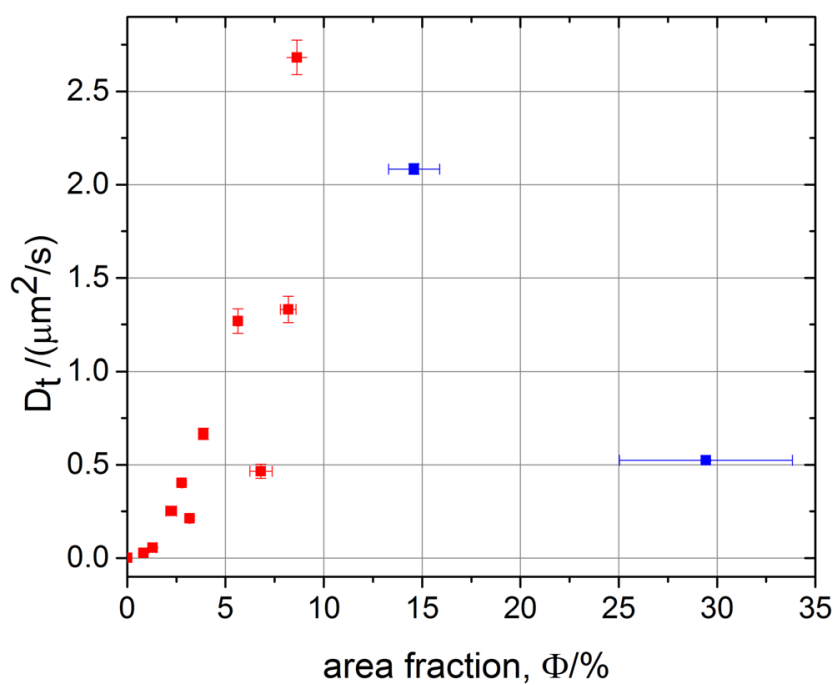

Fig. 7 The translational diffusion constant $D_{\mathrm{t}}$ of elongated silica particles in pure active baths (red symbols) or in active baths with clustering Chlamydomonas reinhardtii (blue symbols) for different values of the area fraction $\Phi$ of algae participating in the dominant interaction with the passive objects. For red symbols, $\Phi$ is the area fraction of motile Chlamydomonas reinhardtii, whereas for the blue symbols $\Phi$ is the area fraction of clustering algae

silica doublet is sufficient to hinder its rotational motion significantly.

For the sparser clustered state, the translational diffusion constant of the passive doublet $D_{\mathrm{t}} \approx 2.1 \mu \mathrm{m}^{2} / \mathrm{s}$ is smaller than the maximum value despite the very high number of swimming algae. The rotational diffusion constant $D_{\mathrm{r}} \approx$ $0.24 \mathrm{rad}^{2} / \mathrm{s}$, in contrast, reaches its maximum among the observed values.

The situations of a particle in a pure active bath or a clustered state with active bath can not be compared easily. The latter case is not well characterised by the area fraction of moving algae. Instead, the area fraction of clustering algae may be a better measure, since the dominating interaction appears to be with the sedimented algae rather than the freely swimming algae. It might still be instructive to combine the data for the translational diffusion constant for both cases. Figure 7 shows the translational diffusion constants for different area fractions of those algae that are involved in the dominant interaction, i.e. either freely swimming or clustering algae. Note that the mere cell concentrations of the data points for the dense clustered state and for the maximum enhancement of diffusion by freely swimming algae are comparable and the data point for the sparser clustered state corresponds to the highest concentration of algae. The translational diffusion constant of the sedimented passive particles does not increase monotonously with the concentration of Chlamydomonas reinhardtii due to the formation of clusters of algae at the lower capillary wall.

\section{Conclusions}

We demonstrated that the motile microalgae Chlamydomonas reinhardtii can enhance the rotational and translational diffusion constants of sedimented elongated silica particles by several orders of magnitude. The concentration dependence of the diffusion constants of the passive particles exhibits a non-monotonic behaviour due to the crowding of sedimented cells at the solid boundary. Crowding of the cells restricts the motion of the passive particles by caging the particles.

In the high-concentration regime, we observe strong short-range positional correlations of the algae forming small clusters. Chlamydomonas reinhardtii were observed to switch between the freely swimming and the clustered state. In both states the motile algae can affect the motion of a passive particle, yet algae in the clustered state also act as a dynamic geometrical restriction.

Acknowledgements The authors acknowledge Prof. Claus-Dieter Ohl and Prof. Ralf Stannarius for fruitful discussions. We thank Dr. Hajnalka Nádasi for assistance in surface treatment and discussions, Dr. Dmitri Puzyrev for assistance with the particle tracking algorithms, Wieland Ivo Schiffermüller for participation in the experiments in the frame of the MINT-Praktikum. The authors also acknowledge technical support by K. Guttmann and Dr. P. Pfeiffer.

Funding information Open Access funding provided by Projekt DEAL. The research was partially supported by Deutsche Forschungsgemeinschaft (Project ER 467/14-1). F.v.R. acknowledges support by a Landesstipendium Sachsen-Anhalt.

\section{Compliance with ethical standards}

Conflict of interest The authors declare that they have no conflict of interest.

Open Access This article is licensed under a Creative Commons Attribution 4.0 International License, which permits use, sharing, adaptation, distribution and reproduction in any medium or format, as long as you give appropriate credit to the original author(s) and the source, provide a link to the Creative Commons licence, and indicate if changes were made. The images or other third party material in this article are included in the article's Creative Commons licence, unless indicated otherwise in a credit line to the material. If material is not included in the article's Creative Commons licence and your intended use is not permitted by statutory regulation or exceeds the permitted use, you will need to obtain permission directly from the copyright holder. To view a copy of this licence, visit http://creativecommons. org/licenses/by/4.0/.

\section{References}

1. Leptos KC, Guasto JS, Gollub JP, Pesci AI, Goldstein RE (2009) Phys Rev Lett 103:198103

2. Kurtuldu H, Guasto JS, Johnson KA, Gollub JP (2011) Proceedings of the National Academy of Sciences 108(26): 10391 
3. Jeanneret R, Pushkin DO, Kantsler V, Polin M (2016) Nat Commun 7:12518

4. Wu XL, Libchaber A (2000) Phys Rev Lett 84:3017

5. Short MB, Solari CA, Ganguly S, Powers TR, Kessler JO, Goldstein RE (2006) Proceedings of the National Academy of Sciences 103(22):8315

6. Katija K (2012) J Exp Biol 215(6):1040

7. Yeomans J (2016) In: Proceedings of the international school of physics Enrico Fermi: soft matter self-assembly, vol 193, p 383

8. Maggi C, Paoluzzi M, Pellicciotta N, Lepore A, Angelani L, Di Leonardo R (2014) Phys Rev Lett 113(23):238303

9. Peng Y, Lai L, Tai YS, Zhang K, Xu X, Cheng X (2016) Phys Rev Lett 116:068303

10. Sokolov A, Apodaca MM, Grzybowski BA, Aranson IS (2010) Proceedings of the National Academy of Sciences 107(3):969

11. Kim MJ, Breuer KS (2007) Anal Chem 79(3):955

12. Yang O, Peng Y, Liu Z, Tang C, Xu X, Cheng X (2016) Phys Rev E 94:042601

13. Mathijssen AJTM, Jeanneret R, Polin M (2018) Phys Rev Fluids 3:033103

14. Gorman D, Levine R (1965) Proceedings of the National Academy of Sciences USA 54:1665

15. Hutner SH, Provasoli L, Schatz A, Haskins C (1950) Proc Am Philos Soc 94(2): 152

16. Highfield R, Thomas R, Cummins P, Gregory D, Mingins J, Hayter J, Schärpf O (1983) In: Langmuir-Blodgett films, 1982. Elsevier, Amsterdam, pp 165-172

17. Tirado MM, Martínez CL, de la Torre JG (1984) J Chem Phys 81(4):2047

18. Kolley F (2017) Experimentelle Untersuchung aktiver und passiver brownscher Dynamik in quasi 2D. Bachelor thesis, Ottovon-Guericke University Magdeburg

19. Leach J, Mushfique H, Keen S, Di Leonardo R, Ruocco G, Cooper JM, Padgett MJ (2009) Phys Rev E 79:026301

20. Pushkin DO, Yeomans JM (2013) Phys Rev Lett 111(18):188101

21. Kreis CT, Le Blay M, Linne C, Makowski MM, Bäumchen O (2017) Nature Physics Advance Online Publication

22. Chaikin PM, Lubensky TC (1995) Principles of condensed matter physics. Cambridge University Press, Cambridge

23. Drescher K, Goldstein RE, Michel N, Polin M, Tuval I (2010) Phys Rev Lett 105:168101

Publisher's note Springer Nature remains neutral with regard to jurisdictional claims in published maps and institutional affiliations.

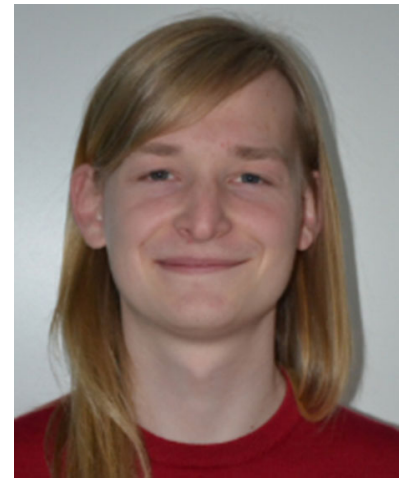

Florian von Rüling received his degree in Physics in 2018 from Otto von Guericke University Magdeburg where he currently is a PhD student. His research topics are biological active matter and freely suspended liquid crystal films.

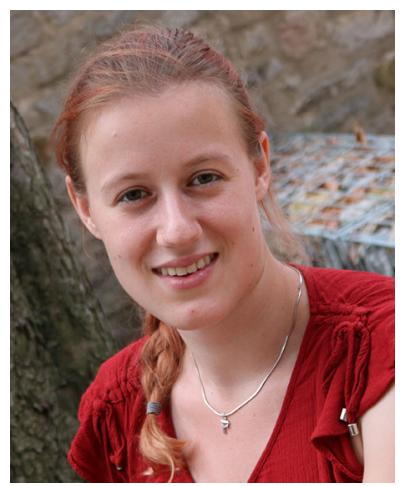

Francine Kolley graduated from Technical University Dresden in 2019. She is currently a $\mathrm{PhD}$ student at the biological algorithms group (cfaed, Dresden) of Benjamin Friedrich. Her research is focused on self-organized pattern formation in complex biological systems.

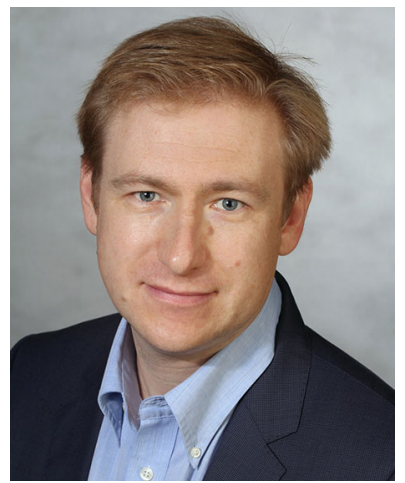

Alexey Eremin graduated in Biophysics from Moscow State University (Russia). In 2003 he received his $\mathrm{PhD}$ at Martin Luther University in Halle/Saale (Germany). He is currently an Associate Professor at Otto von Guericke University in Magdeburg. His research interests are soft matter physics (liquid crystals and colloids), and self-organization in biological systems. 